\title{
Mesh repair for postoperative wound dehiscence in the presence of infection: is absorbable mesh safer than non-absorbable mesh?
}

\author{
M. van't Riet $\cdot$ P. J. de Vos van Steenwijk \\ H. J. Bonjer $\cdot$ E. W. Steyerberg $\cdot$ J. Jeekel
}

Received: 12 June 2006 / Accepted: 19 April 2007 / Published online: 6 June 2007

(C) Springer-Verlag 2007

\begin{abstract}
Objective In patients with postoperative wound dehiscence in the presence of infection, extensive visceral oedema often necessitates mechanical containment of bowel. Prosthetic mesh is often used for this purpose. The aim of the present study was to assess the safety of the use of non-absorbable and absorbable meshes for this purpose. Method All patients that had undergone mesh repair of abdominal wound dehiscence between January 1988 and January 1998 in the presence of intra-abdominal infection were included in a retrospective cohort study. All surviving patients had physical follow-up in February 2001.

Result Eighteen patients were included in the study. Meshes consisted of polyglactin $(n=6)$, polypropylene $(n=8)$, polyester $(n=1)$, or a combination of a polypropylene mesh with a polyglactin mesh on the visceral side $(n=3)$. All patients developed complications, consisting mainly of mesh infection (77\%), intra-abdominal abscess (17\%), enterocutaneous fistula (17\%), or mesh migration through the bowel $(11 \%)$. Mesh removal was necessary in eight patients (44\%). Within four months postoperatively, six patients (33\%) had died because of progressive abdominal sepsis. The incidence of progressive abdominal sepsis was significantly higher in the group with absorbable polyglactin mesh than in the group with nonabsorbable mesh (67 vs. $11 \%, p=0.02$ ) After a mean follow-up of 49 months, $63 \%$ of the surviving patients had developed incisional hernia.
\end{abstract}

M. van't Riet · P. J. d. van Steenwijk · H. J. Bonjer .

E. W. Steyerberg · J. Jeekel $(\square)$

Department of Surgery, University Hospital Rotterdam,

Dr Molewaterplein 40, 3015, GD Rotterdam,

The Netherlands

e-mail: mvantriet@hotmail.com
Absorbable meshes did not yield better outcomes than nonabsorbable meshes in terms of complications and mortality rate.

Conclusion Synthetic graft placement in the presence of intra-abdominal infection has a high risk of complications, regardless of whether absorbable (polyglactin) or nonabsorbable mesh material (polypropylene or polyester) is used, and should be avoided if possible.

Keywords Mesh · Infection · Wound dehiscence . Absorbable $\cdot$ Non-absorbable

\section{Introduction}

Wound dehiscence, defined as postoperative disruption of all layers of the abdominal wall, occurs in $0.25-3 \%$ of all patients after abdominal surgery, and is associated with high morbidity and 10-40\% mortality [1,2]. Intra-abdominal infection is present in up to $40 \%$ of these patients [3].

Patients with postoperative wound dehiscence in the presence of infection or contamination represent a difficult and challenging problem to the surgeon. In these patients, the bowel is often oedematous and protrudes from the abdominal cavity. Evisceration of this oedemateus bowel precludes primary closure of the abdominal wall and necessitates other methods of mechanical control.

Currently, one of the methods most commonly used to mechanically contain the contents of the abdomen in these patients is the use of prosthetic mesh. However, concern exists about mesh-related complications, such as mesh extrusion and enteric fistula formation [4-7]. Several authors have suggested that the occurrence of these complications is particularly associated with the use of nonresorbable mesh and could be prevented through the use of 
an absorbable mesh [8-11]. However, only limited data on this subject are available.

The present study was performed in order to determine the safety of the use of non-absorbable and absorbable meshes for wound dehiscence repair in the presence of infection.

\section{Methods}

In a retrospective study, all patients that had undergone mesh repair of acute postoperative wound dehiscence between January 1988 and January 1998 at the Erasmus University Medical Centre in Rotterdam were selected for analysis [3]. Wound dehiscence was defined as moderate when there was only serosanguinous leakage through the abdominal defect without evisceration. Dehiscence was defined as severe when evisceration had occurred. Evisceration was defined as protrusion of bowel beyond the abdominal wall.

All patients with signs of intra-abdominal infection (defined as intra-abdominal pus and/or a positive bacterial culture from the abdomen) at the time of mesh placement were included in the study. Data regarding patient characteristics, initial surgical procedures, procedure of mesh placement, postoperative complications, microbiological findings, antibiotic therapy and late complications were recorded.

There were no consistent guidelines on the use of mesh and the choice of mesh material. In general, patients with large defects requiring great tension to close were primarily selected for mesh repair. If fascial necrosis was present, necrotectomy was performed before graft placement. If possible, the omentum was placed in-between the mesh and the viscera.

Postoperative mesh infection was defined as discharge of pus from the mesh, confirmed by positive bacterial culture from the mesh. Postoperative progressive abdominal sepsis was defined as progressive sepsis with positive blood cultures of enteric bacteria.

At the time that the study was conducted (February 2001), every surviving patient underwent a physical examination of the abdominal wall at the outpatient department, with special emphasis placed on searching for the presence of incisional hernia. To achieve this, the abdomen was examined in both upright and horizontal positions during the Valsalva manoeuvre. The follow-up period was defined as the time interval between mesh placement and the last physical examination that was performed.

Statistical analysis was performed using the MannWhitney U-test for independent samples. A $p$-value of less than 0.05 was considered to be statistically significant.

\section{Results}

During the study period, a total of 168 patients underwent wound dehiscence repair. Of these, 26 patients had mesh repair of wound dehiscence, of whom 18 patients had repair in the presence of intra-abdominal infection. Thus, eighteen patients (twelve males and six females, with a mean age of 61 years, range 31-89) were included in the study.

The mean number of abdominal operations that preceded the procedure of mesh placement was two (range 1-5). In the patients with wound dehiscence, underlying causes of abdominal infection were gastric perforation $(n=2)$, Boerhaave syndrome $(n=1)$, perforated diverticulitis $(n=3)$, pancreatitis $(n=1)$, contaminated initial procedure with bowel surgery for malignancy or gastrointestinal bleeding $(n=8)$, strangulated bowel in a femoral hernia $(n=1)$, inadvertent gallbladder perforation $(n=1)$ and the occurrence of wound infection after surgery for acute aneurysm of the abdominal aorta $(n=1)$. Severity of dehiscence was moderate in one patient and severe (with evisceration) in 17 patients.

At the time of mesh placement, clinical signs of infection (pus coming out of the wound, temperature elevation and/or high white blood cell count) had been present for a mean of ten days (range 0-22 days). At the time of mesh placement, bacteriaemia (confirmed with positive blood culture) was present in three patients.

Broad-spectrum antibiotic therapy was administered at the start of each surgical procedure in which a mesh was placed. None of the mesh patches was impregnated with antibiotics. Graft materials included polyglactin $(n=6)$, polypropylene $(n=8)$ and polyester $(n=1)$. In another three patients, a polypropylene mesh was combined with a polyglactin mesh on the visceral side. The skin was left open in all patients.

\section{Complications}

Mean postoperative hospital stay was 53 days (range 1-142 days). Postoperative complications and comparison of complications for absorbable or non-absorbable mesh material are shown in Table 1.

Fifteen patients developed mesh infection (77\%), which was bacteriologically confirmed in all cases. A total of 18 different species of pathogens were recovered from the abdominal cavities postoperatively (Table 2). There was no difference between the use of absorbable or non-absorbable mesh in terms of the incidence of mesh infection. Mesh infection was initially treated by broad-spectrum antibiotics in all patients. In eight patients, however, this was not successful and the mesh had to be removed. In three patients, mesh infection was associated with intra-abdominal abscesses, which were surgically drained. 
Table 1 Comparison of postoperative complications between absorbable, non-absorbable and a combination of absorbable plus non-absorbable mesh materials

\begin{tabular}{|c|c|c|c|c|c|}
\hline Complication & $\begin{array}{l}\text { Absorbable } \\
\text { (polyglactin) } \\
(n=6)\end{array}$ & $\begin{array}{l}\text { Non-absorbable } \\
\text { (polypropylene } \\
\text { or polyester) }(n=9)\end{array}$ & $\begin{array}{l}\text { Combination } \\
(\text { polypropylene }+ \\
\text { polyglactin) }(n=3)\end{array}$ & $p$-value & $\begin{array}{l}\text { Total } \\
(n=18)\end{array}$ \\
\hline \multicolumn{6}{|l|}{ Complications } \\
\hline Clinical signs of mesh infection & $67 \%$ & $89 \%$ & $67 \%$ & n.s. & $77 \%$ \\
\hline Enterocutaneous fistula & & $22 \%$ & $33 \%$ & n.s. & $17 \%$ \\
\hline Bowel perforation due to mesh migration & $17 \%$ & $11 \%$ & & n.s. & $11 \%$ \\
\hline Intra-abdominal abscess & $33 \%$ & $11 \%$ & & n.s. & $17 \%$ \\
\hline Ileus & & $22 \%$ & & n.s. & $11 \%$ \\
\hline Urinary tract infection & $17 \%$ & $11 \%$ & & n.s. & $11 \%$ \\
\hline Intra-abdominal bleeding & & & $33 \%$ & n.s. & $6 \%$ \\
\hline Pulmonary complications & $17 \%$ & $11 \%$ & $33 \%$ & n.s. & $17 \%$ \\
\hline Gastro-intestinal bleeding & & $11 \%$ & & n.s. & $6 \%$ \\
\hline Mesh removed & $33 \%$ & $56 \%$ & $33 \%$ & n.s. & $44 \%$ \\
\hline Total mortality & $83 \%$ & $33 \%$ & $33 \%$ & & $56 \%$ \\
\hline \multicolumn{6}{|l|}{ Cause of death } \\
\hline Progressive abdominal sepsis & $67 \%$ & $11 \%$ & $33 \%$ & $p=0.02^{\mathrm{a}}$ & $33 \%$ \\
\hline Cardiopulmonary complications & $17 \%$ & $11 \%$ & & n.s. & $11 \%$ \\
\hline Cerebrovascular accident & & $11 \%$ & & n.s. & $11 \%$ \\
\hline Peritonitis carcinomatosa & & $11 \%$ & & n.s. & $6 \%$ \\
\hline Incisional hernia in surviving patients & $100 \%$ & $67 \%$ & $0 \%$ & n.s. & $63 \%$ \\
\hline
\end{tabular}

n.s. not statistically significant

a comparison between absorbable and non-absorbable materials

Two patients developed enterocutaneous fistulas. In the first patient, two enterocutaneuous fistulas had developed five months after placement of a combined polypropylene with polyglactin mesh. In the second patient, the fistula was diagnosed 18 months after the use of a polyester mesh. In both patients, the mesh was removed and partial bowel resection was performed.

In two other patients, the mesh had migrated through and thus perforated the bowel. An absorbable polyglactin mesh had been used in one of these patients. Despite removal of the mesh with partial bowel resection and several relaparotomies with abscess drainage, this patient died at 71 days postoperatively due to progressive abdominal sepsis. In the other patient, a polypropylene mesh had migrated through the bowel and recurrent wound dehiscence had occurred. In this patient, the mesh was removed and a partial bowel resection was performed successfully.

In four patients, after mesh removal, a new mesh composed of polypropylene $(n=3)$ or ePTFE (ethylpolytetrafluoroethylene, $n=1$ ) was placed into the defect. Of these patients, two were reoperated and had their mesh removed again. Reasons for mesh removal in these patients were the presence of an enterocutaneous fistula at nine months after placement of a polypropylene mesh, and infection of an ePTFE mesh, which did not respond to antibiotic therapy.
Postoperatively, two patients developed ileus. In one patient, the ileus could be treated successfully with conservative therapy, but in the other patient reoperation was indicated. In this patient, adhesiolysis of dense bowel adhesions to a polypropylene mesh was performed.

Six patients died due to progressive abdominal sepsis (at a range of 1-126 days postoperatively). The incidence of this complication was significantly higher in the group with absorbable polyglactin mesh than in the group with nonabsorbable mesh (67 vs. $11 \%, p=0.02$, Table 1$)$.

By the time that the study was conducted ten patients had died (Table 1). All patients who were still alive underwent physical examinations at the outpatient department, except for one patient who could not be traced (13\%). After a mean follow-up of 49 months (range 8-133 months), five of these patients had developed incisional hernia (63\%, Table 1).

\section{Discussion}

As shown by the present study, prosthetic mesh placement in patients with wound dehiscence in the presence of intraabdominal infection has a high risk of complications, regardless of whether non-absorbable or absorbable mesh is used. 
Table 2 Pathogens recovered from the abdomen

\begin{tabular}{lc}
\hline Pathogen & $\begin{array}{l}\text { No. of patients with } \\
\text { these pathogens }\end{array}$ \\
\hline Aerobic pathogens & \\
Escherichia coli & 5 \\
Enterococcus sp. & 8 \\
Staphylococcus aureus & 4 \\
Staphylococcus epidermidis & 3 \\
B-Hemolytic streptococcus & 1 \\
Corynebacterium sp. & 2 \\
Acinobacter sp. & 4 \\
Proteus sp. & 12 \\
Pseudomonas sp. & 1 \\
Streptococcus sp. & 7 \\
Enterobacter sp. & 4 \\
Klebsiella sp. & 1 \\
Bacillus sp. & 3 \\
Morganella morganii & 3 \\
Serratia marcescens & \\
Anaerobic pathogens & 1 \\
Bacteroides sp. & \\
Mycosis & \\
Candida sp. & \\
\hline
\end{tabular}

Polypropylene mesh is not absorbable and is the material most widely used for abdominal wall replacement and reinforcement during hernia repair. Favourable characteristics of polypropylene include its durability, pliability, high tensile strength, and good growth of fibroblasts into the mesh $[12,13]$. Further, some authors have suggested that if the polypropylene mesh gets infected, this can generally be treated adequately with drainage and antibiotics, without the need for removal of the mesh $[12,14]$. However, this was not found in the present study, in which $56 \%$ of the meshes had to be removed, despite antibiotic therapy. In addition, as shown in the present study, the use of polypropylene mesh in a contaminated environment is associated with a high incidence of serious complications, such as mesh migration through the bowel $(11 \%)$, ileus due to adhesion of bowel to the mesh (11\%), and enteric fistulation (22\%). This was also found by other authors who noted a fistula rate of $12-50 \%$ [4,15-18].

Polyglactin and polyglycolic acid meshes are both rapidly absorbable. They can temporarily restore abdominal wall continuity, but when the mesh has been absorbed, all patients will inevitably develop incisional hernia, as confirmed by the present study $[8,11,19,20]$.

Several authors have suggested that the use of absorbable meshes would reduce the occurrence of mesh-related chronic complications [8-11]. However, as shown by the present study, the use of absorbable polyglactin mesh was associated with an incidence of mesh migration through the bowel that was comparable to that of polypropylene mesh (17 vs. 11\%). Further, enterocutaneous fistulas still developed despite placement of a polyglactin mesh on the visceral side of a polypropylene mesh. In addition, the incidence of mortality due to persistent abdominal sepsis was even higher in the group of patients with absorbable polyglactin meshes compared to non-absorbable polypropylene meshes. Since this is a retrospective study, there may be a bias in patient selection. However, a factor that may contribute to the high incidence of progressive abdominal sepsis with the use of absorbable polyglactin mesh is the multifilament structure of this mesh compared to the monofilament structure of the polypropylene mesh. It is known that multifilament material is more susceptible to infection than monofilament material, and the use of a multifilament foreign body in an infected environment may increase bacterial load on the mesh [21-23].

Several authors have attempted to develop alternatives for the temporary containment of abdominal contents in the presence of large contaminated abdominal wall defects that do not use prosthetic mesh [24-28]. Ghimenton et al. used an empty, sterile, 1- or 3- litre plastic bag, as used for intravenous fluid administation or for urological irrigation, which was stitched with a continuous suture to the edges of the rectus sheath or the skin [25]. When no relaparotomy was needed, the plastic bag was removed and split skin grafting was performed. However, all surviving patients developed incisional hernias, which were demanding to repair. Further, massive bowel adherence to the broad midline granulation area with skin graft still posed a small risk of fistula formation, which was seen in 2 out of 75 patients [25].

Koniaris et al. described the "dynamic retention technique" [24]. With this technique, a bowel bag is used to cover the bowel and omentum. Moistened burn dressings are placed flatly over the bowel bag, and four or five horizontal retention sutures are placed over this dressing, on top of which a second layer of dressings is added with a drainage catheter. In the ICU, retention sutures may be tightened and delayed primary fascial closure can be achieved. No fistulas were seen and only one out of ten patients developed incisional hernia [24].

Recently, a new device was developed for temporary abdominal closure, the $\mathrm{VAC}^{\circledR}$ abdominal dressing. With this technique, foam that is encapsulated within a non-adhesive layer is placed over the bowel and covered with a drape. This is connected to a vacuum suction system that creates a local negative pressure on the wound, thereby enabling evacuation of abdominal fluids. Since the nonadhesive layer is placed under the fascia, the bowel cannot adhere to the fascia and so the surgeon can approximate the fascia after several days if the intra-abdominal swelling is reduced. Although first results with this device are promising, its use requires further study and evaluation $[29,30]$. 
Another alternative is to use biological meshes. These meshes, composed of small intestinal submucosa (SIS), porcine dermal collagen, bovine pericardium or human acellular tissue, become an integral part of the body. They are advocated by some authors for use in operations with a high risk of infection because they provide the specific advantage of resistance to superficial wound infections [31-33]. However, these biological mesh implants are fairly costly and additional clinical studies are required to evaluate their efficacy.

In conclusion, the present study shows that synthetic graft placement in the presence of intra-abdominal infection has a high risk of complications, regardless of whether absorbable polyglactin mesh or non-absorbable polypropylene or polyester mesh is used. Use of absorbable mesh material was even associated with a significantly increased incidence of progressive abdominal sepsis compared to non-absorbable mesh material. Therefore, use of "conventional" mesh under contaminated circumstances should be avoided if possible, and alternatives such as the dynamic retention method, VAC abdominal dressing closure and use of biological meshes should be explored. Prospective randomised trials are required to determine the best approach to use in order to manage large infected abdominal wall defects.

\section{References}

1. Riou JPA, Cohen JR, Johnson H (1992) Factors influencing wound dehiscence. Am J Surg 163:324-330

2. Muckart JM, Luvuno FM (1985). Abdominal wound disruption. J R Coll Surg 30:47-49

3. Van 't Riet M, De Vos van Steenwijk PJ, Bonjer HJ, Steyerberg EW, Jeekel J (2004) Incisional hernia after repair of wound dehiscence: incidence and risk factors. Am Surg 70:281-286

4. Voyles CR, Richardson JD, Bland KI, Tobin GR, Flint LM, Polk HC (1981) Emergency abdominal wall reconstruction with polypropylene mesh. Ann Surg 194:219-23

5. Stone HH, Fabian TC, Turkleson ML, Jurkiewicz MJ (1981) Management of acute full-thickness losses of the abdominal wall. Ann Surg 193:612-618

6. Boyd WC (1977) Use of Marlex mesh in acute loss of the abdominal wall due to infection. Surg Gynecol Obstet 144:251-252

7. Wouters DB, Krom RAF, Slooff MJH (1983) The use of Marlex mesh in patients with generalized peritonitis and multiple organ system failure. Surg Gynecol Obstet 156:909-914

8. Buck JR, Fath JJ, Siu-Keung Chung MPH, Sorensen VJ, Horst HM, Obeid FN (1995) Use of absorbable mesh as an aid in abdominal wall closure in the emergent setting. Am Surg 61:655-658

9. Porter JM. (1995) A combination of Vicryl and Marlex mesh: a technique for abdominal wall closure in difficult cases. J Trauma 39:1178-1180

10. Dayton MT, Buchele BA, Shirazi SS, Hunt LB (1986) Use of an absorbable mesh to repair contaminated abdominal wall defects. Arch Surg 121:954-960

11. Greene MA, Mullins RJ, Malangoni MS, Feliciano PD, Richardson JD, Polk HC Jr (1993) Laparotomy wound closure with absorbable polyglycolic acid mesh. Surg Gynaecol Obstet $176: 213-218$
12. Mc Neeley SG jr, Hendrix SL, Bennett SM, Singh A, Ransom SB, Kmak DC, Morley GW (1998) Synthetic graft placement in the treatment of fascial dehiscence with necrosis and infection. Am J Obstet Gynaecol 179:1430-1435

13. Brandt CP, McHenry CR, Jacobs DG, Piotrowski JJ, Priebe PP (1995) Polypropylene mesh closure after emergency laparotomy: morbidity and outcome. Surgery 118:736-741

14. Turkcapar AG, Yerdel MA, Aydinuraz K, Bayar S, Kuterdem E (1998) Repair of midline incisional hernias using polypropylene grafts. Surg Today 28:59-63

15. Kendrick JH, Casali RE, Lang NP, Read RC (1982) The complicated septic abdominal wound. Arch Surg 117:464-468

16. Gilsdorf RB, Shea MM (1975). Repair of massive septic abdominal wall defects with Marlex mesh. Am J Surg 130:634-638

17. Jones JW, Jurkovich GJ (1989) Polypropylene mesh closure of infected abdominal wounds. Am Surg 55:73-76

18. Nagy KK, Fildes JJ, Mahr C, Roberts RR, Frosner SM, Joseph KT, Barrett J (1996) Experience with three prosthetic materials in temporary abdominal wall closure. Am Surg 62: 331-335

19. Emmink B, Thomson SR, Moodley M, Muckart DJJ (1993) Laparotomy closure using perisplenic polyglactin mesh. J R Coll Surg Edinb 38:177-178

20. Levasseur JC, Lehn E, Rignier P (1979) Repair of extensive eviscerations using an absorbable prosthesis. J Chir 116:737-739

21. Osther PJ, Gjode P, Mortensen BB, Mortensen PB, Bartholin J, Gottup F (1995) Randomized comparion of polyglycolic acid and polyglyconate sutures for abdominal fascial closure after laparotomy in patients with suspected impaired wound healing. Br J Surg 82:1080-1082

22. Stol DW (1978) De invloed van hechtmaterial of de wondgenezing. Thesis, Davids Decor, Alblasserdam

23. Wissing JC (1988) Het sluitstuk van de laparotomie. Een proscpectief gerandomiseerd multicentre onderzoek naar de resultaten van fasciesluiting. Thesis, Helmond, Wibrodissertatiedrukkerij

24. Koniaris LG, Hendrickson RJ, Drugas G, Abt P, Schoeniger LO (2001) Dynamic retention. A technique for closure of the complex abdomen in critically ill patients. Arch Surg 136:1359-1363

25. Ghimenton F, Thomson SR, Muckart DJ, Burrows R (2000) Abdominal content containment: practicalities and outcome. Br J Surg 87:106-109

26. Smith PC, Tweddell JS, Bessey PQ (1992) Alternative approaches to abdominal wound closure in severely injured patients with massive visceral edema. J Trauma 32:16-20

27. Bender JS, Bailey CE, Saxe JM, Ledgerwood AM, Lucas CE (1994). The technique of visceral packing: recommended management of difficult fascial closure in trauma patients. J Trauma 36:182-185

28. Sherck J, Seiver A, Shateney C, Oakes D, Cobb L (1998) Covering the open abdomen: a better technique. Am Surg 64:854-857

29. Miller PR, Thompson JT, Flaer BJ, Meredith JW, Chang MC (2002) Late fascial closure in lieu of ventral hernia: the next step in open abdomen management. J Trauma 53:843-849

30. Erdmann D, Drye C, Heller LBSN, Wong MS, Levin LS (2001) Abdominal wall defect and enterocutaneous fistula treatment with the vacuum-assisted closure (VAC system). Plast Reconst Surg 108:2066-2068

31. Catena F, Ansaloni L, Gazzotti F, Gagliardi S, Di Saverio S, D'Alessandro L, Pinna AD (2007) Use of porcine dermal collagen (Permacol) for hernia repair in contaminated fields. Hernia 11:5760

32. Alaedeen DI, Lipman J, Medalie D, Rosen MJ (2007) The singlestaged approach to the surgical management of abdominal wall hernias in contaminated fields. Hernia 11:41-45

33. Patton JH Jr, Berry S, Kralovich KA (2007) Use of human acellular dermal matrix in complex and contaminated abdominal wall reconstructions. Am J Surg 193:360-363 